\title{
Axial Disc Motor Experimental Analysis Based in Steinmetz Parameters
}

\author{
David Inácio ${ }^{1}$, João Martins ${ }^{1}$, Mário Ventim Neves ${ }^{1}$, Alfredo Álvarez ${ }^{2}$, \\ and Amadeu Leão Rodrigues ${ }^{1}$ \\ ${ }^{1}$ CTS/UNINOVA, Faculty of Sciences and Technology - University New of Lisbon, \\ Quinta da Torre, 2829-516 Caparica, Portugal \\ ${ }^{2}$ Department of Electrical Engineering, Escuela de Ingenierias Industriales, \\ Universidad de Extremadura, E-06006 Badajoz, Spain
}

\begin{abstract}
Nowadays an economical and environment crisis is felt in the world due to the increasing fuel prices and high $\mathrm{CO}_{2}$ emissions. This crisis is mostly due to present the transportation system, which uses internal combustion engines. The development and integration of electrical motors with improved electro mechanical characteristics, using high temperature superconductors, can provide a sustainable future replacing the conventional internal combustion motors.

An axial type disc motor, with high temperature superconductor (HTS) material has been developed and tested in order to obtain an electrical equivalent circuit based on the experimental results. The tested HTS motor exhibits a conventional hysteresis motor type of behavior, even though the hysteretic phenomena don't have the same principle. The proposed approach allows the description of the equivalent electrical circuit as a conventional hysteresis motor.
\end{abstract}

Keywords: Axial disc motor; HTS materials; YBCO.

\section{Introduction}

Economical, environmental and political issues make the optimization and improvement of electric machines necessary to develop electrical vehicles and ensure a sustainable future. These types of vehicles are developed purely electric (for example, batteries or fuel cell and hydrogen fed) or hybrid (combustion and electric fed), being integrated in various projects.

The HTS materials present some advantages making them unique. Almost null DC resistivity, high current transportation capability and trapping flux capability, enables increasing efficiency several electrical applications [1-4]. These advantages allow the developing of superconducting electrical machines with higher specific torque than their conventional counterparts [5], [11-12].

Electrical machines with HTS bulk rotor present a complex behavior, showing both synchronous and asynchronous regimes [5], [7]. Even though they are similar to conventional hysteresis motors, their operating principle is different. It is appropriate to study the behavior of superconducting hysteresis motors in asynchronous regime using Steinmetz-type models, in order to compare it with the conventional induction motor and to clarify the effect of the HTS bulk material in this regime. 
In this paper, the relations between the equivalent circuit's parameters and the motor's characteristics are discussed, for a conventional induction disk motor and for a HTS hysteresis disk motor, composed by a rotor with a polycrystalline YBCO disk [12]. This will be made using the Steinmetz-type "T" model.

\section{Contribution to Sustainability}

The paper presents an analysis of two types of disc motors: conventional and high temperature superconducting. This analysis, based on experimental parameters and electromechanical characteristics, allows to conclude that the HTS disc motor presents better electromechanical characteristics that the conventional one. The use of HTS disc motors in future full electric vehicles, based on fuel cell power supplies, will contribute to ensure the desired full electric vehicles levels of environmental sustainability.

\section{Expression of Equivalent Electrical Circuit}

The steady state per phase Steinmetz electrical equivalent circuit of both a HTS hysteresis and an induction motor, considering that the stator's winding number of turns is equal to the rotor's winding number of turns, can be expressed as shown in fig. 1 (see notation in Appendix A2) [8]. Eddy current component was neglected.

In both motors the rotor resistance is separated as a component proportional to the electrical losses, $R_{\mathrm{A}}$, and as a component proportional to the mechanical output, $R_{\mathrm{B}}$, shown in fig. 1.

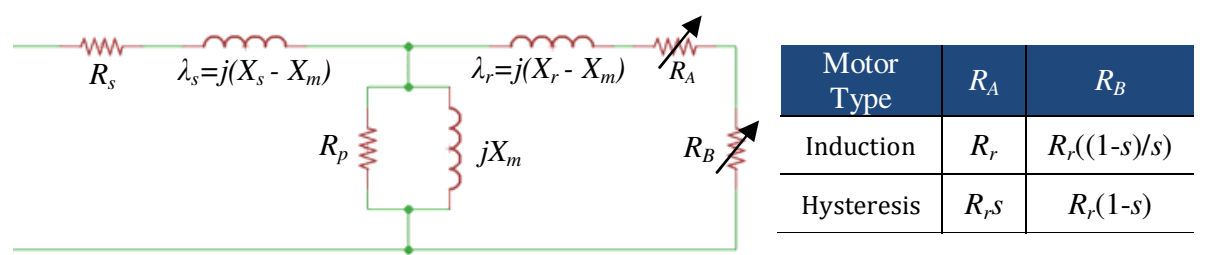

Fig. 1. Equivalent Steinmetz's electrical circuit for a conventional induction and hysteresis motor [8]

To completely specify the Steinmetz equivalent circuit one has to establish its parameters. For the motor equivalent electrical circuit, the stator circuit parameters can be easily determined, directly in the stator. However this could not be done on the rotor side.

$$
\begin{aligned}
& \lambda_{s}=(1-\alpha k) X_{s}=0,33 X_{s} \\
& \lambda_{r}=\left(\alpha^{2}-\alpha k\right) X_{s}=0,33 X_{s} \\
& X_{m}=\alpha k X_{s}=0,67 X_{s}
\end{aligned}
$$

For the induction motor, these parameters are estimated through the blocked-rotor and no-load tests. For the HTS hysteresis motor, according to [8] and for $\alpha=1$ (value for 
a squirrel-cage type induction motor) and $\mathrm{k}=0.67$ (calculated in [8] for a similar motor), Steinmetz's parameters are given by equation (1).

\subsection{Blocked-Rotor, No-Load and Load Tests}

For blocked-rotor test the mechanical speed is null, hence $s=1$, and therefore $R_{B}=0$ (for both induction and hysteresis machines), which corresponds to a "virtual" shortcircuit in the rotor's side equivalent circuit. At no-load test the mechanical speed is equal to the synchronous speed, hence $s=0$, leading to a rotor's side open circuit for the induction motor. In each test, the stator winding voltage $U$, the winding current $I$, and the electrical power $P_{\text {elect }}$ are measured per phase. The per phase complex impedance is given by $\bar{Z}=Z \cdot e^{j \varphi}$, where $Z=U / I$ and $\varphi=\cos ^{-1}\left[P_{\text {elect }} /(U . I)\right]$. Longitudinal and transversal complex impedances are, respectively, obtained using the blockedrotor test $(s=1)$, where the magnetization current is neglecting, and the no-load test $(s=0)$, where the stator impedance is neglecting. Both are presented in (2)

$$
\overline{Z_{L}}=R_{\text {L.eq }}+j X_{L . e q}\left\{\begin{array}{l}
R_{L . e q}=\left|\overline{Z_{L}}\right| \cdot \cos (\varphi)=R_{s}+R_{r} \\
X_{L . e q}=\left|\overline{Z_{L}}\right| \cdot \sin (\varphi)=X_{s}+X_{r}
\end{array} ; \overline{Z_{T}}=R_{m}+j X_{m}\left\{\begin{array}{l}
R_{m}=\left|\overline{Z_{T}}\right|(\cos (-\varphi))^{-1} \\
X_{m}=\left|\overline{Z_{L}}\right|(\sin (-\varphi))^{-1}
\end{array}\right.\right.
$$

Determining the value of $R_{\mathrm{s}}$ by independent measurements, and considering $X_{s}=X_{r}$ (which is a usual assumption, based on theoretical considerations and supported on practical evidence [13]), all the parameters of Steinmetz's equivalent electrical circuit can be obtained from the previous relations.

The load test is performed with the aim of studying the motor's behavior. It consists in applying some mechanical load torque, driven by the shaft. From the analysis and computation of torque and speed measurements the motor's mechanical characteristics could be obtained.

\section{Power Output and Torque Analysis}

Even though HTS materials present a different hysteresis phenomenon, when compared with the ferromagnetics ones, in both of them, AC losses are directly proportional to the hysteresis loop area and to the frequency. The output power and the electromechanical torque, in a HTS machine, are given in table 1 (appendix A1).

Table 1. HTS Hysteresis motor and Induction motor electromechanical characteristics

\begin{tabular}{|c|c|c|}
\hline $\begin{array}{l}\text { HTS Hysteresis } \\
\text { Machine }\end{array}$ & $P_{\text {mec }}=P_{\text {elect }}(1-s)=P_{H / \text { cicle }} \cdot p \cdot f_{s}(1-s)$ & $T_{\text {elmec }}=\frac{p^{2}}{2 \pi} P_{H / \text { cicle }}$ \\
\hline Induction Machine & $P_{m e c}=R_{c}^{\prime} \cdot I_{r}^{2}=R_{r}^{\prime}\left(\frac{1-s}{s}\right) \cdot I_{r}^{2}$ & $T_{\text {elmec }}=\frac{p}{\omega_{\text {sup }}} \frac{R_{r}^{\prime}}{s}$ \\
\hline
\end{tabular}

The conventional induction machine is analyzed, according the most literature, based in the Steinmetz equivalent circuit. The power output and torque equations are disposed in table 1, that permits conclude that, for the HTS motor, the output power is 
proportional to the AC losses in the HTS material and to the slip, which means that for values of speed near of synchronous speed, all the AC losses in HTS materials is transformed in mechanical output power.

The analysis of table 2 allows to conclude that, for the HTS motor, the output power is proportional to AC losses in the HTS materials and to the sleep, this means that close to synchronism operation, all HTS materials AC losses are converted into mechanical output power. The electromechanical torque is independent of the slip and directly proportional to HTS AC losses, with a factor of $2 \pi$ (this result is in line with [5] and [8]). Higher HTS material AC losses imply higher mechanical power and torque.

\section{Experimental Results}

\subsection{Test Method and Experimental Apparatus}

In this paper the blocked-rotor, no-load and load tests were performed for a conventional induction motor. The blocked-rotor and load tests were only made for the HTS motor with the main objective of finding the Steinmetz's parameters and observing the behavior of both conventional and superconductor motors.
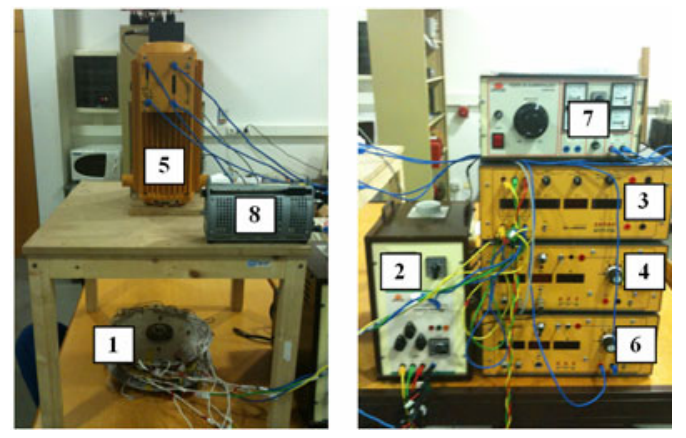

Fig. 2. Experimental apparatus for induction and HTS hysteresis motor's tests

In the performed tests the motors $\{1\}$ were fed with 3-phase [phase-to-phase rms voltage of $40 \mathrm{~V}$ (conventional induction motor) and $23 \mathrm{~V}$ (HTS motor)], four poles and a $50 \mathrm{~Hz}$ supply frequency configuration. The mechanical load was obtained by means of a DC generator (controlled by two dc power supply and a control resistance) driven by the motors' shaft and feeding a resistive load. The basic used instrumentation is depicted in fig. 2: a transformer $\{2\}$ to feed the motor; a commercial electrical $\{3\}$ and mechanical $\{4\}$ power measuring modules to measures the electrical and mechanical parameters in the system. The DC machine $\{5\}$ had sensors, which communicate with the modules, to mechanical torque and speed measurement. The DC Generator was controlled with two DC current supply $\{6\}\{7\}$ and a control resistance $\{8\}$. During the superconductor motor's tests, only the disk motor was immersed in liquid nitrogen. Different values of electrical current is due to limitations in the supply transformer, whose rated current is limited to $20 \mathrm{~A}$. 


\subsection{Experimental Determination of Steinmetz Equivalent Electrical Circuit}

For the presented induction motor, using the equations presented in (2) and from the measured experimental results, in table 2, the parameters were determined. For the HTS hysteresis motor the measured electric quantities were used to compute the longitudinal complex impedance and using (1), the Steinmetz parameters were computed. All the computed values are presented in table 2.

Table 2. Stator's measured and obtained Steinmetz's parameters

\begin{tabular}{c|cc|ccccc}
\cline { 2 - 7 } & \multicolumn{3}{c|}{ Measured results } & \multicolumn{5}{c}{ Computed results } \\
\hline & $R_{s}[\Omega]$ & $X_{s}[\Omega]$ & $\lambda_{s}, \lambda_{r}[\mathrm{mH}]$ & $R_{r}[\Omega]$ & $R_{m}[\Omega]$ & $X_{m}[\Omega]$ & $\mid$ \\
\hline Induction Machine & 1,06 & 0,47 & 1,50 & 0,028 & 2,85 & 2,2 & $\mid$ \\
HTS Hysteresis Machine & 0,3 & 0,27 & 0,088 & 0,0008 & 2,85 & 0,18 \\
\hline
\end{tabular}
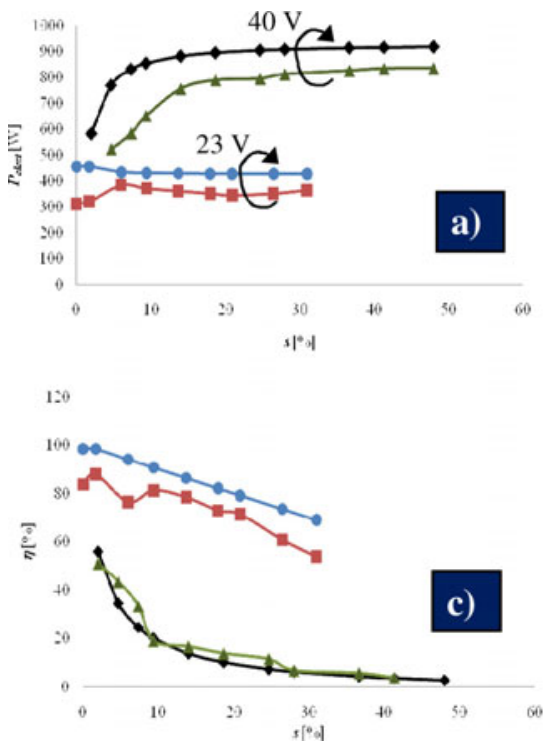

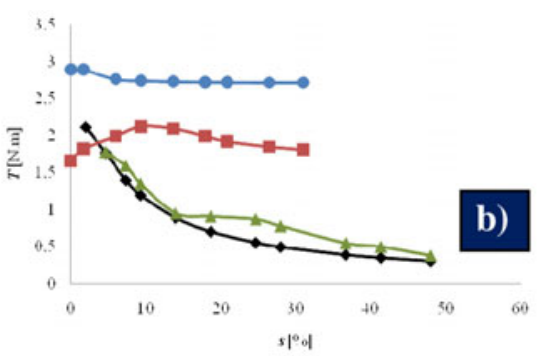

$\rightarrow$ Conventional Motor - Theoretical Results. $\mp$ Conventional Motor - Experimental Results $\leadsto-$ HTS Motor - Theoretical Results.

HTS Motor - Expenimental Results.

Fig. 3. Comparison between theoretical and experimental a) electrical power, b) developed torque and c) efficiency of conventional induction and HTS hysteresis motor

The value of the magnetic losses, $R_{m}$, was calculated based upon the induction motor case and considered the same for the both cases. For the HTS case, doesn't exist information that allows its computation.

The load test was performed as described in section 3. For both motors, the theoretical electromechanical characteristics were computed, based on the experimentally obtained parameters and using the theoretical analysis above described. These were compared with the experimentally obtained characteristics in tests. Various slip-dependent characteristics were present in figure 3: a) one can see that the electrical power is higher than the correspondent value in HTS motor, which 
is expected because the supply voltage is different; b) and c) respectively, shows the both motor's torque and the efficiency. The experimental characteristics have a trend evolution coherent with the theoretically predicted ones.

The comparison between predicted and measured quantities must be taken as being essentially indicative, for two reasons. On one hand, the measured quantities, being much smaller than the measuring apparatus' ranges, suffer from an important relative error. And, on the other hand, the predicted quantities refer to the internal power or torque electrodynamically developed, while the measured ones refer to the available quantities in the motor's shaft, which differ from the first ones by the mechanical losses. The viscosity friction in the liquid nitrogen and the friction in the conventional bearings working at low temperature cause non negligible losses when compared with the low quantities developed. Therefore, the measured results for the HTS motor are consistently and significantly lower than the theoretical ones.

\section{Conclusions and Future Work}

The research \& development in the electrical machinery and control areas could provide innovative machines that associated to innovative control methods can be used to achieve a desirable sustainable future. The integration of HTS materials provides better performances in the electrical machines.

Low power prototypes of a conventional induction axial type motor and a HTS axial type motor were tested. The conventional induction motor presents a higher range of measured slip values than the HTS hysteresis motor because with a $30 \%$ slip value the current was near to the maximum that the system supply could deliver. Nevertheless the minimum value of slip is high. To obtain more detailed initial values the synchronism speed test must be performed in future work.

The torque ratio between the HTS and the conventional motors isn't as higher as expected. The different supply voltage used can be the reason for the shown behavior. Still, the HTS motor presents a higher efficiency compared with the induction motor. However, further research work must be done to explain the obtained results, with the implementation of an optimized experimental apparatus that includes a supply transformer allowing higher current and including a measurement system with a range adequate for the measured values.

Acknowledgements. Authors would like to thank to CTS of UNINOVA and to FCT (CTS multiannual funding - through the PIDDAC Program funds) for the financial support for this work.

\section{References}

1. Kawabata, S., Nakahama, Y., Kawagoe, A., Sumiyoshi, F.: Development of a Compact HTS Current Transformer for Evaluating the Characteristics of HTS Conductors. IEEE Transactions on Applied Superconductivity 18(2), 1147-1150 (2008)

2. Noe, M., Juengst, K.-P., Werfel, F., Cowey, L., Wolf, A., Elschner, S.: Investigation of high-Tc bulk material for its use in resistive superconducting fault current limiters. IEEE Transactions on Applied Superconductivity 11(1), 1960-1963 (2001) 
3. Nomura, S., Tanaka, N., Tsuboi, K., Tsutsui, H., Tsuji-Iio, S., Shimada, R.: Design considerations for SMES systems applied to HVDC links. In: 13th European Conference on Power Electronics and Applications, EPE 2009, pp. 1-10, 8-10 (2009)

4. Ohsaki, H., Tsuboi, Y.: Study on electric motors with bulk superconductors in the rotor. Journal of Materials Processing Technology 108, 148-151 (2001)

5. Barnes, G., Dew-Hughes, D., McCulloch, M.: Finite difference modelling of bulk high temperature superconducting cylindrical hysteresis machines. Supercond. Sci. Technol. 13(2), 229-236 (2000)

6. Inácio, D., Martins, J., Ventim-Neves, M., Álvarez, A., Leão-Rodrigues, A.: Disc motor: Conventional and superconductor simulated results analysis. In: Camarinha-Matos, L.M., Pereira, P., Ribeiro, L. (eds.) DoCEIS 2010. IFIP Advances in Information and Communication Technology, vol. 314, p. 505. Springer, Heidelberg (2010)

7. Inacio, D., Inacio, S., Pina, J., Valtchev, S., Ventim-Neves, M., Martins, J., LeãoRodrigues, A.: Conventional and HTS disc motor with pole variation control. In: Power Engineering, Energy and Electrical Drives, POWERENG 2009, vol. 18, p. 513 (2009)

8. Jung, H., Nakamura, T., Tanaka, N., Muta, I., Hoshino, T.: Characteristic analysis of hysteresis-type $\mathrm{Bi}-2223$ bulk motor with the use of equivalent circuit. Physica $\mathrm{C}$ : Superconductivity 405(2), 117-126 (2004)

9. Wilson, M.: Superconducting Magnets. Oxford Science Publications (1983)

10. Sharma, N., Bedford, R.: Hysteresis Machines, Mumbai, India (2003)

11. Tsuboi, Y., et al.: Torque Characteristics of a Motor Using Bulk Superconductors in the Rotor in Transient Phase. IEEE Trans. Appl. Supercond. 13, 2210 (2002)

12. Inácio, D., Pina, J., Gonçalves, A., Ventim-Neves, M., Leão-Rodrigues, A.: Numerical and Experimental Comparison of Electromechanical Properties and Efficiency of HTS and Ferromagnetic Hysteresis Motors. In: 8th European Conference on Applied Superconductivity (EUCAS 2007), Brussels, Belgium (2007)

13. Ventim-Neves, M.: Máquina Assíncrona. Notes of Electrotécnia Teórica, Internal publication, FCT-UNL

\section{Appendix}

\section{A1 - Power Output and Torque Analysis: HTS Hysteresis Motor}

According to [9], and since the applied magnetic field is equal to the full penetration field [10], the AC losses in the HTS materials are proportional to the hysteresis loop and frequency, as

$$
P_{\text {AC }}=P_{H / \text { cicle }} \cdot f_{\text {mag }},
$$

where $P_{H / c i c l e}$ is given approximately by $\xi \cdot H_{\text {appl }}^{2}$ and $\xi$ is a factor depending on the characteristics and geometry of the superconductor. Assuming that the applied load torque is less than the motor torque, the motor accelerates until it reaches the synchronism, during the sub-synchronous regime the frequency of the rotor's magnetization is given by $f_{r o}=p\left(f_{s s}-f_{m e c}\right)=p . f_{s s} . s . f_{s s}$ is the mechanical synchronous speed, in revolutions per second (if $f_{\text {sup }}$ is the supply's frequency, in $\mathrm{Hz}$, then $f_{\mathrm{s}}=f_{\text {sup }} / p$ ). To each value of the frequency $f$, there is a corresponding angular speed $\omega=2 \pi f$. The frequency $f_{r o}$ is the frequency of the induced currents in the 
rotor - in this case, in the HTS material - and therefore is the magnetization frequency in (3). The AC losses power are, therefore, given by

$$
P_{A C}=P_{H / c i c l e} \cdot p \cdot f_{s} \cdot s,
$$

The mechanical power, for the energy conservation principle, neglecting power and electric losses, results

$$
P_{\text {mec }}=P_{\text {elect }}-P_{H / \text { cicle }} \cdot p \cdot f_{s} \cdot s .
$$

At the motor starting, the slip is 1 and the mechanical speed is zero, so $P_{m e c}=0$. Replacing in (6) gives

$$
P_{\text {elect }}=P_{H / \text { cicle }} \cdot p \cdot f_{s} \Rightarrow P_{\text {mec }}=P_{\text {elect }}(1-s) .
$$

From (6) it is possible to observe that when the rotor accelerates from the start until the synchronous speed, the hysteresis power losses decreases, the developed mechanical power increases and the electrical power is kept constant.

The mechanical torque, $T_{\text {elmec }}$, is given by

$$
T_{\text {elmec }}=P_{\text {mec }} / \Omega_{\text {mec }} \Rightarrow T_{\text {elmec }}=\frac{p^{2}}{2 \pi} P_{H / \text { cicle }} .
$$

With the mechanical speed given by

$$
\Omega_{\text {mec }}=\omega_{s} \cdot(1-s)=\frac{2 \pi \cdot f_{\text {sup }}}{p} \cdot(1-s)
$$

\section{A2 - Notations}

\begin{tabular}{|l|l|}
\hline$R$ & Resistance \\
\hline$X$ & Reactance \\
\hline$\lambda$ & leakage reactance \\
\hline subscript $s$ & Stator \\
\hline subscripts $r$ & Rotor \\
\hline subscripts $m$ & Mutual/magnetization \\
\hline subscripts $p$ & Iron \\
\hline subscripts $c$ & Load \\
\hline Indice & Referred to stator \\
\hline$P$ & Power per phase \\
\hline subscript elect & Electric \\
\hline subscript $A C$ & Alternating current \\
\hline subscript $m e c$ & Mechanical \\
\hline subscript $H /$ cicle & hysteresis losses per cycle \\
\hline
\end{tabular}

\begin{tabular}{|l|l|}
\hline$H$ & Magnetic field \\
\hline subscript appl & Applied \\
\hline$f$ & Frequency \\
\hline subscript mag & Magnetization \\
\hline subscript $s s$ & Synchronous speed \\
\hline subscript sup & Supply \\
\hline subscript mec & $\begin{array}{l}\text { Rotor's mechanical } \\
\text { speed }\end{array}$ \\
\hline subscript ro & Rotor's induced currents \\
\hline$T$ & torque \\
\hline subscript elmec & Electromechanical \\
\hline$p$ & Number of poles pair \\
\hline$\Omega_{m e c}$ & mechanical speed \\
\hline
\end{tabular}

